DOI: $10.35643 /$ Info.26.1.15

Artículo original

\title{
Marcos de Datos de Feminicidio. Reconstrucción ontológica y análisis crítico de dos datasets de asesinatos de mujeres por razones de género
}

\author{
Data Frames of Feminicide. An ontological reconstruction and critical \\ analysis of activist and government datasets of gender-related murders of \\ women \\ Quadros de dados do feminicídio. Reconstrução ontológica e análise crítica \\ de dois conjuntos de dados de assassinatos de mulheres por razões de gênero
}

Helena Suárez Val ${ }^{\mathrm{a}}$

a Estudiante doctoral, Centre for Interdisciplinary Methodologies, University of Warwick, Reino Unido. ORCID: 0000-0002-8748-0734. Correo electrónico: helena.suarez-val@warwick.ac.uk.

\section{Resumen}

El feminicidio (o femicidio) es una categoría feminista que designa -y denuncia- a las muertes violentas de género de mujeres, adolescentes y niñas, en especial sus asesinatos. En América Latina, la categoría ha proporcionado un marco para que las activistas feministas y, más recientemente, los estados recopilen datos sobre el feminicidio. Este trabajo busca comprender las implicancias de las formas en que se estructuran, clasifican y curan los datos de feminicidio, como estos arreglos dirigen las acciones posibles. A través de tres ideas clave -el feminicidio como marco, las trazas digitales de asesinato y los marcos de datos-, el estudio propone un abordaje teórico y metodológico para el análisis de la organización y la presentación de datos sobre asesinatos de mujeres relacionados con el género. Utilizando el método de reconstrucción ontológica, el estudio examina dos datasets de asesinatos de mujeres por razones de género en Uruguay, producidos por una activista y por el estado. Este trabajo muestra cómo las descripciones que proponen los datasets habilitan ciertas acciones (y otras no) y concluye con recomendaciones para revisar el diseño de los datasets de feminicidio y para futuras líneas de investigación.

Palabras clave: FEMINICIDIO; ESTUDIOS DE DATOS; POLÍTICA ONTOLÓGICA.

\section{Abstract}


Feminicide (or femicide) is a feminist category that designates -and denouncesthe gender-related violent deaths of women, adolescents, and girls, especially murders. Especially in Latin America, the category has provided a frame for feminist activists, and most recently governments, to gather data about feminicide. This work seeks to understand the implications of how feminicide data are structured, sorted, and curated, how these arrangements guide potential actions. The study advances an approach for the analysis of the organisation and presentation of data about gender-related murders of women by developing three key ideas: feminicide as a frame, digital traces of murder, and data frames. With ontology reconstruction as a method, the study analyses an activist and a government dataset of gender-related murders of women in Uruguay. The study shows how the descriptions provided in each dataset enable certain actions (and not others) and concludes by providing recommendations for reviewing the design of these datasets and for future research avenues.

Keywords: FEMINICIDE, DATA STUDIES, ONTOLOGICAL POLITICS.

\section{Resumo}

Femicídio (ou femicídio) é uma categoria feminista que designa - e denuncia - as mortes violentas de mulheres, adolescentes e meninas, principalmente seus assassinatos. Na América Latina, a categoria forneceu uma estrutura para ativistas feministas e, mais recentemente, estados para coletar dados sobre o feminicídio. Este trabalho busca compreender as implicações das formas como os dados do femicídio são estruturados, classificados e curados, como esses arranjos direcionam possíveis ações. Por meio de três ideias-chave - referencial do feminicídio, vestígios digitais de homicídio e frames de dados - o estudo propõe uma abordagem teórica e metodológica para a análise da organização e a apresentação de dados sobre assassinatos de mulheres relacionados ao gênero. Usando o método de reconstrução ontológica, o estudo examina dois conjuntos de dados de assassinatos de mulheres por razões de gênero no Uruguai, produzidos por um ativista e pelo Estado. Este trabalho mostra como as descrições propostas pelos conjuntos de dados possibilitam certas ações (e outras não) e conclui com recomendações para revisar o desenho dos conjuntos de dados do feminicídio e para futuras linhas de pesquisa.

Palavras-chave: FEMINICÍDIO, ESTUDOS DE DADOS, POLÍTICA ONTOLÓGICA.

Fecha de recibido: $21 / 10 / 2020$

Fecha de aceptado: 28/12/2020

\section{Introducción}

El feminicidio (o femicidio) es una categoría feminista que designa -y denuncialas muertes violentas por razones de género de mujeres, adolescentes y niñas, en 
especial sus asesinatos. Desde la construcción de la violencia doméstica como problema social y público, hasta la más reciente movilización del término feminicidio, las feministas han inventado diversos conceptos "con el fin político de reconocer y visibilizar la discriminación, la opresión, la desigualdad y la violencia sistemática contra la mujer que, en su forma más extrema, culmina en la muerte" (Bernal Sarmiento et al., 2014, p. 13). La categoría entonces sirve para reconocer ciertas muertes violentas de mujeres y su movilización sirve para denunciar el contexto amplio de violencia por razones de género, al cual contribuyen, según Lagarde (2008, p. 217), “el silencio social, la desatención, la idea de que hay problemas más urgentes y la vergüenza y el enojo que no conminan a transformar las cosas sino a disminuir el hecho y demostrar que no son tantas "las muertas"'. Contra este contexto, el desarrollo de la categoría dio lugar a una nueva ecología de atención (Bowker \& Star, 1999, p. 267), cambiando la desatención por la memoria, la indiferencia por la acción.

Para las mujeres y las activistas feministas latinoamericanas, esta categoría generó un marco colectivo para la acción, incluyendo acciones que combinan formas tradicionales de movilización con aquellas típicas del activismo de datos (Chenou \& Cepeda-Másmela, 2019, p. 397). Llevar un registro de la violencia contra las mujeres "eleva el fenómeno de anécdota a categoría, conduciendo a su mayor visibilidad" (Osborne, 2008, p. 101). Por tanto, gran parte del trabajo de denuncia de la categoría se realiza a través de prácticas de datos: la recopilación y circulación de datos sobre casos que encajan en el marco de feminicidio. Feminicidio Uruguay (FU), un registro digital de casos de feminicidio que inicié en 2015, es un ejemplo de este tipo de "feminismo de datos" (D’Ignazio \& Klein, 2020), donde el monitoreo de medios, el registro y la circulación de datos online van de la mano de manifestaciones callejeras, performances públicas, cabildeo y otras formas de acción contra esta forma de violencia.

Por otra parte, todos los países de América Latina (excluyendo Cuba) cuentan con leyes que integran variantes de la categoría, como femicidio, feminicidio y/o violencia feminicida (Deus \& González, 2018, p. 34). En Uruguay, en 2017 y 2018, respectivamente, se aprobaron una ley que define el femicidio como agravante del homicidio (Ley N. ${ }^{\circ}$ 19538) y otra más amplia donde se define la 
"violencia femicida" entre otros tipos de violencias basadas en género (Ley N. 19580). Entre otras obligaciones, esta última requiere que el estado genere datos cuantitativos y cualitativos sobre la violencia basada en género (Parlamento del Uruguay, 2018, Ley N. ${ }^{\circ}$ 19580, Art. 11 J). En marzo de 2019, el Ministerio del Interior del Uruguay (MI) publicó en el portal estatal Catálogo de Datos Abiertos un dataset titulado Seguridad y violencia basada en género, integrando datos de femicidio. Este dataset constituye un ejemplo de cómo los estados pueden movilizar los datos abiertos contra la violencia (Fumega, 2019).

Recientemente, se ha resaltado necesidad de realizar investigaciones que analicen cómo los datos se estructuran, ordenan y curan[1] en arreglos socio-técnicos que dan forma a lo que se vuelve visible, cognoscible y pasible de acción (Flyverbom \& Murray, 2018, p. 2). En la presente coyuntura, esta investigación propone entender la implicación de los diferentes arreglos socio-técnicos de datos en la amplificación de ciertos aspectos de los asesinatos de mujeres - o cómo los distintos arreglos de datos dan lugar a diferentes versiones de los asesinatos de mujeres por razones de género.

\section{Situando la investigación}

El objetivo de este trabajo es explorar cómo la categoría da un marco para el diseño de las estructuras de datos y cómo éstas enmarcan a los asesinatos por razones de género, sus circunstancias y actores implicados, estructurando y condicionando las posibles interpretaciones del fenómeno $\mathrm{y}$, por ende, las respuestas y acciones afectivas y políticas.

Al poner el foco en cómo se organizan y presentan los datos, este trabajo se alinea con los Estudios Críticos de Datos (critical data studies), que entienden que la tecnología nunca es neutral y los datos nunca crudos[2] y que tienen un interés particular en los contra-datos creados por activistas y movimientos sociales (Dalton \& Thatcher, 2014; D’Ignazio \& Klein, 2020; Kitchin \& Lauriault, 2014; Milan \& Velden, 2016). El marco analítico se nutre de los estudios feministas sobre el feminicidio, del análisis de las categorías y las prácticas de categorización realizado desde los estudios de infraestructura (Bowker \& Star, 1999) y los estudios de ciencia y tecnología y la filosofía de la ciencia (Hacking, 
1999; Mol, 1999) y de las nociones de marco y enmarcar desarrolladas en las ciencias sociales y las humanidades (Butler, 2009; Entman, 1993; Minsky, 1974). El trabajo no busca resolver las tensiones y las superposiciones entre estos enfoques, sino más bien aprovechar sus aportes complementarios para arrojar luz sobre los materiales de investigación.

Si bien hay un creciente interés académico sobre los datos de feminicidio, incluyendo su medición e indicadores (por ejemplo Dawson \& Carrigan, 2020; Vives-Cases et al., 2016; Walby et al., 2017; Weil et al., 2018), hay pocas investigaciones que examinen directamente los datos y las prácticas de datos que se producen frente al fenómeno. Algunos ejemplos incluyen el examen crítico de las estadísticas de violencia de género en México de Tavera Fenollosa (2008); el análisis antropológico de Engle Merry y Bibler Coutin (2014) sobre tecnologías de medición de la violencia contra las mujeres; el trabajo de Chenou y CepedaMásmela (2019) sobre \#NiUnaMenos y el activismo de datos "desde el sur"; mis propios trabajos sobre mapeadoras de feminicidio y sobre datos discordantes en Uruguay (Suárez Val, 2017, 2020); y una investigación-acción sobre feminicidio y prácticas de datos activistas que estamos desarrollando Data+Feminism Lab (MIT), ILDA y FU. Dada la actual crisis de feminicidio en América Latina, es crucial continuar construyendo conocimientos sobre los datos y las prácticas de datos emergentes tanto desde el activismo como los estados.

Si, como propuso Hacking (1991, pp. 254-255), las personas actúan y toman decisiones con arreglo a las descripciones, ¿Qué posibilidades diferentes para la descripción, y por tanto para la acción, habilitan los arreglos de datos sobre asesinatos de mujeres que producen las activistas y los estados? ¿Cómo se arregla[3] (Bowker \& Star, 1999) los datos para encajar estos asesinatos en el marco, promoviendo así, como indica Entman (1993, p. 52), una definición del problema, una interpretación causal, una evaluación moral y/o una recomendación de tratamiento particulares? ¿Qué políticas ontológicas (Mol, 1999) se vislumbran en los diferentes marcos de datos? En las siguientes secciones presento un análisis de la organización y la presentación de datos sobre asesinatos de mujeres por razones de género, guiado por tres concepciones: el feminicidio como marco, las trazas digitales de asesinato y los marcos de datos. El trabajo utiliza el método de 
reconstrucción ontológica (de Vos, 2014; de Vos et al., 2012) para examinar cómo las activistas y el estado en Uruguay organizan y presentan las trazas digitales de los asesinato en tablas, rastreando las entidades y relaciones constituidas (Hacking, 1983/2002)[4] en estos arreglos para elucidar qué acciones se habilitan. La conclusión ofrece algunas recomendaciones para refinar estos marcos de datos, con la esperanza de contribuir a la lucha para acabar con la violencia contra las mujeres.

\section{EI feminicidio como marco (categórico)}

El término feminicidio es una categoría creada y movilizada por las académicas y activistas feministas para nombrar y denunciar, como expresaron las mujeres argentinas en huelga en 2016, “el punto más alto de una trama de violencias, que anuda explotación, crueldades y odio a las formas más diversas de autonomía y vitalidad femeninas, que piensa nuestros cuerpos como cosas a usar y descartar, a romper y saquear" (Ni Una Menos, 2016, sec. III). La palabra feminicidio, traducción de Lagarde (2010) del inglés femicide (Radford \& Russell, 1992), ha unido a las activistas feministas latinoamericanas en protesta contra ésta y toda forma de violencia contra las mujeres a lo largo y ancho del continente. Según datos mundiales, en 2018, 14 de los 25 países con las tasas más altas de femicidio/feminicidio se encontraban en América Latina y el Caribe (Ruíz \& Garrido, 2018). Tomando la discusión de Stengers y Ralet (1997, pp. 216-217) sobre un "evento del SIDA", podría decirse que América Latina está viviendo un “evento del feminicidio", en la medida que la categoría se inscribe cada vez más en el registro político y ético, se intenta plantear el problema con claridad y la disputa sobre qué expertos/as se reconocen legítimos interlocutores articula a actores en el activismo, la academia, los medios y los estados. En suma, la categoría feminicidio proporciona un marco para la acción y un marco para diferenciar los asesinatos de mujeres, entre relacionados al género o no.

El Modelo Protocolo para la Investigación de Femicidio/Feminicidio en América Latina de las Naciones Unidas define el feminicidio como:

la muerte violenta de mujeres por razones de género, ya sea que tenga lugar dentro de la familia, unidad doméstica o en cualquier otra relación interpersonal, en la comunidad, por parte de cualquier persona, o que sea 
perpetrada o tolerada por el Estado y sus agentes, por acción u omisión (Bernal Sarmiento et al., 2014, p. 14)

Hablar de muertes violentas por razones de género implica entender que, como expresan Walby y colegas (2017, p. 42), el género satura todas las instituciones sociales y no es externo a la violencia, sino que estructura las características centrales del evento, que es un tipo de relación social entre el agresor y la víctima. Aunque aún foco de lo que Goffman (1974/1986) denomina "disputas de marco" -sobre los significados y el alcance exactos del término y sus solapamientos con femicidio y otras categorías relacionadas como femigenocidio (Segato, 2011), feminicidio sexual serial o sistémico (Monárrez Fragoso, 2009, 2018), o travesticidio/transfeminicidio (Bento, 2014; Berkins, 2015; Maffía, 2016)-[5], el término ha proporcionado un marco organizador, aportando "coherencia discursiva a los múltiples contextos nacionales en los que las mujeres son asesinadas debido a su género" (Bueno-Hansen, 2011, p. 386).

En términos amplios, las activistas feministas han superado (si no resuelto) sus diferencias, adoptando uno o los dos términos y adaptando su definición al país, contexto, campaña u objetivo del cabildeo (Manjoo, 2012, p. 7). Asimismo, a pesar de las "controversias penales" (Toledo Vásquez, 2009, p. 62) que ha provocado, todos los países de América Latina (excepto Cuba) cuentan con legislación que penaliza el femicidio/feminicidio (Deus \& González, 2018, p. 34). La categoría (con todos sus matices) ha contribuido a estimular intervenciones tanto activistas como estatales para abordar esta forma de violencia, amén de proporcionar un marco para poner en evidencia la prevalencia del feminicidio (Deus \& González, 2018, pp. 12-13).

Orientadas políticamente por emociones feministas y motivadas por generar cambios sociales y/o exigir políticas públicas, las activistas han creado marcos para recopilar, presentar y circular datos sobre los casos (ver Suárez Val, 2017). Por su parte, con diferentes (y superpuestas) definiciones y motivaciones, los estados también producen y publican datasets sobre la violencia, en parte como parte del ejercicio de vigilancia del poder burocrático, que depende del conocimiento de la población para vigilar a la sociedad y mantener el orden (Poster, 1995, p. 91). La producción de estos datos es un acto profundamente 
político y contendido y las disputas sobre cuáles muertes contar en qué categorías resultan en datos discordantes (Suárez Val, 2020), pudiendo llevar a una "guerra de cifras" entre y dentro de las instituciones estatales, las organizaciones de la sociedad civil y otros actores (Tavera Fenollosa, 2008, p. 302).

Independientemente de la definición categórica, cada caso en sí puede ser reconocido como un evento: un antes y después de la muerte violenta de una mujer. Según Stengers (2000, pp. 67-68), la medida de un evento es objeto de múltiples interpretaciones, pero también puede medirse por la multiplicidad misma de estas interpretaciones, sólo la indiferencia da "prueba" de los límites del alcance del evento. Afectadas (de formas diferentes) por las muertes violentas de mujeres, las activistas y los estados responden recopilando y presentando datos producto de interpretaciones de los eventos sustentadas en sus respectivos marcos categóricos, que vienen con ciertos dados ontológicos (Butler, 2009, p. 149), incluyendo las concepciones de género, poder, violencia, mujeres, hombres, etc. (Luján Pinelo, 2018). Pero en las prácticas de datos en particular, como sugieren Gitelman y Jackson (2013, p. 37), los datos probablemente no tengan ninguna relación con la verdad o la realidad más allá de la realidad que ellos mismos nos ayudan a construir. La producción de datos de feminicidio, entonces, no sólo constituye una política de enmarcar, sino también una práctica de política ontológica donde la realidad no precede a las prácticas mundanas en las que interactuamos con ella, sino que se moldea dentro de estas prácticas (Mol, 1999, p. 75).[6] Y aunque, como indica Fraser (2010, p. 65), es imposible elaborar una lista de las entidades que entran en un evento con antelación porque las identidades y las relaciones adquieren definición a través del propio evento, no obstante en sus prácticas de datos las activistas y los estados hacen precisamente eso, al diseñar ontologías de datos que describen e implican - es decir, que ponen en la escena de los asesinatos por razones género- (id)entidades y relaciones específicas.

\section{Trazas digitales de asesinato}

Obtener y recopilar datos es indispensable para conocer la magnitud, forma y comportamiento de la violencia letal de género y para desarrollar respuestas 
públicas adecuadas (Tavera Fenollosa, 2008, p. 303; ver también Vives-Cases et al., 2016; Walby et al., 2017). En consecuencia, tanto las activistas como los estados recopilan y presentan públicamente datos sobre los casos que encajan en el marco de feminicidio. En estos trabajos, los criterios para el arreglo de datos sobre los casos, para organizar y presentar los datos, se sustentan (restringen o activan) en la forma en que los activistas o los estados conciben al feminicidio como categoría, así como también en las habilidades, los formatos y las tecnologías disponibles, como ser las bases de datos o las hojas de cálculo y sus tablas. El término trazas digitales[7] refiere a los casi ubicuos procesos de datificación: el proceso de convertir las actividades humanas en datos para darles usos múltiples (Flyverbom \& Murray, 2018, p. 1; ver también Reigeluth, 2014). Sin embargo, bien podría aplicarse a los procesos (más artesanales (ILDA, 2019, p. 2)) mediante los cuales la actividad humana de asesinar a una mujer se convierte en datos que luego se usan para coordinar protestas callejeras, para el cabildeo o para formular políticas públicas.

Para Segato (2004, p. 7), los asesinatos de mujeres por razones género son, en última instancia, una expresión de control sobre la voluntad de otra persona que se enuncia en dos ejes de comunicación. Verticalmente, el asesino se dirige a una mujer, pero en el plano horizontal, el feminicidio es el discurso del asesino hacia sus propios pares, una promesa de entrar en la "hermandad viril" que asegura y afirma la masculinidad (Segato, 2004, p. 7). En esta geometría, el paso fatal de un feminicida en la vida de una mujer deja una secuencia de huellas que reverberan sobre los dos ejes, hacia el cuerpo social. Esta secuencia se (re)imprime en noticias, informes policiales, fallos judiciales y publicaciones en redes sociales sobre los casos, que las activistas feministas y los funcionarios públicos[8], guiados por diferentes marcos, escudriñan en busca de rastros. Siguiendo la conceptualización de Reigeluth (2014, p. 249), utliizo el término trazas digitales de asesinato para referir a las huellas, a los mínimos indicios, a las marcas que deja el evento, traducidos a datos almacenados en bases de datos, mapas y tablas.

Estas trazas digitales de eventos violentos saturados de género se enmarcan en la categoría feminicidio (o no) y se curan, organizan y ponen en acción para acabar con la violencia contra las mujeres. Y, nos dicen Pine y Liboiron (2015, p. 3149), 
esos datos no son solo políticos en el sentido de que se pueden utilizar para la gobernanza; también son políticos en el sentido de que ejercen un poder político encubierto al traer determinados elementos a las tablas e infraestructuras de datos. Las tablas e infraestructuras de datos, entonces, son un sitio donde el feminicidio como categoría y la experiencia vivencial, empírica y situada de los asesinatos de mujeres se co-construyen o convergen (Bowker \& Star, 1999, p. 193).

\section{Marcos de datos}

En su libro Frames of War (Marcos de guerra), Butler (2009, p. 7,8) afirma que no hay vida ni muerte sin relación a algún marco y que la forma de organizar y presentar un hecho conduce a una conclusión interpretativa sobre el hecho en sí, un proceso de enmarque que no es solo cognitivo, sino también afectivo (Coleman \& Banning, 2006). El campo de la inteligencia artificial nos permite pasar de la categoría como marco, al marco como estructura de datos para representar una situación estereotipada (Minsky, 1974). Desde este ángulo, los marcos de datos (data frames) son estructuras organizadas que expresan los valores de características que dan definición a entidades o categorías: grupos de casillas en las que se introducen los valores de variables definidas (Klein et al., 2006, p. 90). Entendidas como marcos, las estructuras de datos definen, conectan y filtran los datos (Klein et al., 2006, p. 89) y de esta manera definen lo que importa (y lo que no) y guían nuestra atención (Flyverbom \& Murray, 2018, p. 10)[9]. Es importante señalar que los datos deben entenderse como enmarcados y enmarcantes (Gitelman \& Jackson, 2013, p. 5), por lo que hay (al menos) dos operaciones superpuestas. Por un lado, las activistas y los estados abordan los asesinatos de mujeres a través de marcos categóricos (feminicidio u otros) que guían su atención mientras investigan cada caso y deciden si encaja, dando forma y definición a los datos relevantes (Klein et al., 2006, p. 88). Por otro lado, las trazas digitales resultantes se presentan en tablas: marcos de datos en forma de cuadrículas de casillas donde ciertos elementos resultan más o menos prominentes (y otros se omiten), así guiando la atención de quienes las leen.

Las tablas donde se arreglan las trazas digitales de los "hechos", su disposición vertical y horizontal reminiscente del modelo gráfico de feminicidio de Segato, 
son marcos por excelencia. Estos marcos de datos, como los marcos categóricos a través de los que comprendemos o, de hecho, no logramos comprender las vidas de otros como perdidas o heridas (como perdibles o heribles), están políticamente saturados (Butler, 2009, p. 1). La noción de política ontológica implica que las diferentes herramientas para manipular un objeto no revelan diferentes aspectos del objeto, sino que realizan diferentes versiones del mismo (Mol, 1999, p. 83). Y si bien las diferentes versiones de los asesinatos de mujeres que promulgan los marcos de datos activistas o del estado pueden ser discordantes, también pueden colaborar e incluso depender entre sí (Mol, 1999, p. 83). Por esto es importante entender las políticas ontológicas en juego, cómo diferentes entidades y relaciones se ponen en escena través de arreglos específicos de trazas digitales de asesinato en marcos de datos.

Quienes diseñan marcos de datos -asignando, ordenando y nombrando las casillas- poseen conocimiento del dominio, es decir saben cuáles son los objetos y procesos importantes, cómo se pueden definir y cómo se relacionan en términos de estructura y causalidad (de Vos, 2014, p. 2). Activistas y funcionarios utilizan su conocimiento del dominio de la violencia, modulado por los matices específicos de la categoría con la que trabajan, para diseñar las tablas que enmarcarán trazas digitales de asesinatos de mujeres: cada fila delinea un caso, cada columna propone un descriptor para las entidades y relaciones implicado en el caso, cada casilla contiene una traza de evidencia específica. Así, realidades desordenadas se enmarcan y estabilizan para que puedan circular en el mundo como unidades homogéneas (Pine \& Liboiron, 2015, p. 3150). Al incluir en la ontología de datos determinados elementos y propiedades, se eleva su prominencia, haciendo que una pieza de información resulte más notoria, significativa o memorable para el público (Entman, 1993, p. 53). Al dirigir la atención hacia, y realizar, diferentes versiones de los asesinatos de mujeres: feminicidio, homicidio doméstico, asesinato "normal"... los distintos marcos de datos regulan las disposiciones afectiva y éticas hacia la violencia, a través de su enmarque selectivo y diferencial (Butler, 2009, p. 1).

\section{Metodología}


El objetivo de este estudio es analizar lo marcos de datos, entendidos como un lugar de convergencia entre el feminicidio como categoría y la actividad humana de asesinar mujeres. Trazas digitales de estos casos se configuran en estructuras de datos complejas, marañas socio-técnicas (Flyverbom \& Murray, 2018, p. 6) que implican a categorías; marcos legales; activistas feministas; investigaciones policiales; noticias; funcionarios públicos; bases de datos; plataformas; entidades y relaciones; y, también, tablas. Analizar esta constelación está más allá del alcance de este trabajo, por esta razón, se coloca el foco en las ontologías de datos de las tablas de las activistas y del estado en que se organizan las trazas digitales de los asesinatos de mujeres.

La pregunta metodológica que se hace este trabajo es, ¿cuáles son las entidades y relaciones que se promulgan en arreglos específicos para la recolección y transmisión de datos diseñados para encajar en el marco del feminicidio? ¿Qué podemos decir sobre los asesinatos de mujeres a partir del arreglo de las casillas en una tabla? ¿Qué entidades y relaciones encajan en el marco (de datos), dándoles prominencia? ¿Qué descripciones, y por tanto acciones, hacen disponibles? Con el fin de analizar las ontologías que movilizan los datasets de FU y MI llevé a cabo una reconstrucción ontológica de cada dataset, método desarrollado por de Vos y colegas (2012, sec. 1) para inferir el modelo conceptual de un proyecto de investigación a partir de la implementación de sus hojas de cálculo o tablas.

Los esquemas de clasificación son muy difíciles de discernir y analizar (Gitelman \& Jackson, 2013, pp. 8-9) y es difícil desenmarañar e incluso hablar de las lógicas que intervienen en el proceso de diseño de las estructuras de datos (Flyverbom \& Murray, 2018, p. 6). La primera tarea fue examinar los títulos de los trabajos, cómo se presentan en línea y otros elementos que "enmarcan el marco". A continuación, buscando elucidar qué entidades y relaciones se ponen en la escena en cada tabla, escudriñé las columnas, considerando tanto los valores, términos y fórmulas, como su ubicación relativa y absoluta (de Vos et al., 2012, sec. 3) y prestando especial atención a las etiquetas, considerando que la elección de nombres en estructuras sintácticas o de código es una pista clara y, a veces, fuerte del marco subyacente (Pan \& Kosicki, 1993, pp. 62-63). Finalmente, visualicé las 
ontologías reconstruidas usando un lenguaje gráfico desarrollado para diagramas entidad-relación (Chen, 1976). En el análisis que sigue, delineo las ontologías de datos de cada dataset, identificando los elementos más prominentes, trazando la alineación de las ontologías reconstruidas con el modelo conceptual de feminicidio que las informa y examinando críticamente las descripciones e interpretaciones, y por lo tanto las acciones, que hacen disponibles.

\section{Reconstrucción ontológica}

\subsection{Feminicidio Uruguay}

A fines de 2014, siendo miembro de la comisión "Feministas en Alerta y en las Calles" de la Coordinadora de Feminismos UY, participé en el inicio de un registro colectivo de casos de feminicidio en Uruguay, que luego continué de forma independiente como Feminicidio Uruguay (FU). Este proyecto se puede situar en una larga genealogía de "nombrar y mostrar" la violencia por razones de género (La República de las Mujeres, 2018). En 1989, el suplemento La República de las Mujeres del Diario La República, comenzó el apartado Crónica de la violencia, una "primera cuantificación" que continuó hasta que la Dirección Nacional de Prevención Social del Delito del MI comenzó un registro oficial de la violencia hacia las mujeres (La República de las Mujeres, 2018, 2018). Desde el activismo, el primer registro de datos públicamente visible sobre muertes violentas de mujeres por razones de género en Uruguay fue la campaña Crespón Negro, que iniciaron en 2001 las mujeres de la Comuna Mujer del Centro Comunal Zonal 9 (Labastie, 2007, p. 27). En 2003, Haydée Gallegos puso este registro a disposición en el sitio web de la ONG Caminos, con el nombre Quienes eran[10], donde siguió incorporando casos hasta mediados de 2014. En 2017, con permiso de Haydée, sumé el registro de Caminos a la base de datos FU y, siguiendo una investigación de Sofía Vanoli Imperiale (2014), agregué asesinatos de mujeres trans, logrando así un registro unificado (aunque seguramente incompleto) de casos de feminicidio desde 2001 hasta la fecha.

El marco de datos de FU inicialmente consistía en una tabla de Google Spreadsheets, que luego visualicé en un mapa con ZeeMaps[11]. Klein, Moon y Hoffman $(2006$, p. 88) han propuesto que si los marcos dan forma y definen los 
datos relevantes, los datos a su vez exigen que los marcos cambien de forma. Aunque los autores se refieren a marcos cognitivos, con el paso del tiempo los datos de FU exigieron modificaciones estructurales del marco de datos. Algunos casos problematizaron el diseño de la tabla, la cantidad de casos sumada a problemas técnicos dictó el traslado de ZeeMaps a Google Maps y finalmente fue necesario crear un marco más amplio para sostener y circular el proyecto: un sitio web[12] y cuentas en redes sociales (Facebook[13], Twitter[14], e Instagram[15]). Los datos de los casos están organizados y se presentan en una hoja de cálculo de Google titulada "Feminicidio en Uruguay desde marzo 2001" y un Google Maps, ambos incrustados en el sitio web mediante la función HTML < iframe> (marco interactivo). La hoja de cálculo integra en una sola tabla los datos actuales, datos históricos del registro colaborativo iniciado en 2014 y datos del memorial de Caminos, preservando así trazas digitales de casos desde marzo de 2001. El mapa consiste en datos copiados de la hoja de cálculo y pegados en "tablas de datos" Google Maps, una por año desde 2014, cada una constituyendo una capa de marcadores.

Cada página del sitio web enmarca los datos con un encabezado sobre el $<$ iframe $>$ que repite los hashtags \#Feminicidio \#MachismoMata \#NiUnaMenos y una definición:

Feminicidio: muertes violentas de mujeres por razones de género, mujeres de dis tintas partes del país, de distintas edades, con distintas historias y planes de vida. Mujeres que hoy no están aquí.

El tono del texto sugiere el encuadre afectivo, enfatizado en la página "Acerca de" donde se declara que el dataset se actualiza "con dolor y rabia" con cada nuevo caso reportado en las noticias. Como se verá, son estos elementos, es decir el enmarque del marco de datos, más que la tabla en sí, los que guían la atención con más fuerza hacia el feminicidio como marco para los asesinatos que presenta FU.

¿Qué (id)entidades y relaciones pone en la escena del feminicidio el marco de datos de FU? ¿A qué versión de los asesinatos de mujeres dan lugar? El siguiente diagrama visualiza la reconstrucción ontológica realizada a partir del análisis del arreglo de trazas digitales (Figura 1). Algunas entidades se nombran explícitamente, otras se señalan indirectamente, otras sólo pueden percibirse a 
través de sus propiedades. El título ofrece un marco temporal, "desde marzo de 2001", la columna denominada "Fecha" marca el ritmo, cada fila un marco congelado del continuo carrete de casos de feminicidio. El título también demarca una ubicación geográfica amplia, "Uruguay", cada caso localizado con mayor precisión bajo la etiqueta "Lugar", ubicación que se reafirma con valores para coordenadas GPS ("Latitud" y "Longitud") y para divisiones territoriales administrativas y políticas ("Departamento" y "País"). Aquí se puede ver al Estado justo entrando al marco, la prominencia dada a divisiones administrativas reconociendo (y tal vez, reforzando) una distribución específica de poder estatal. La entidad Persona se vislumbra apenas a través de las primeras etiquetas que designan sus propiedades "Nombre" y "Edad".

Figura 1: Reconstrucción ontológica de FU.

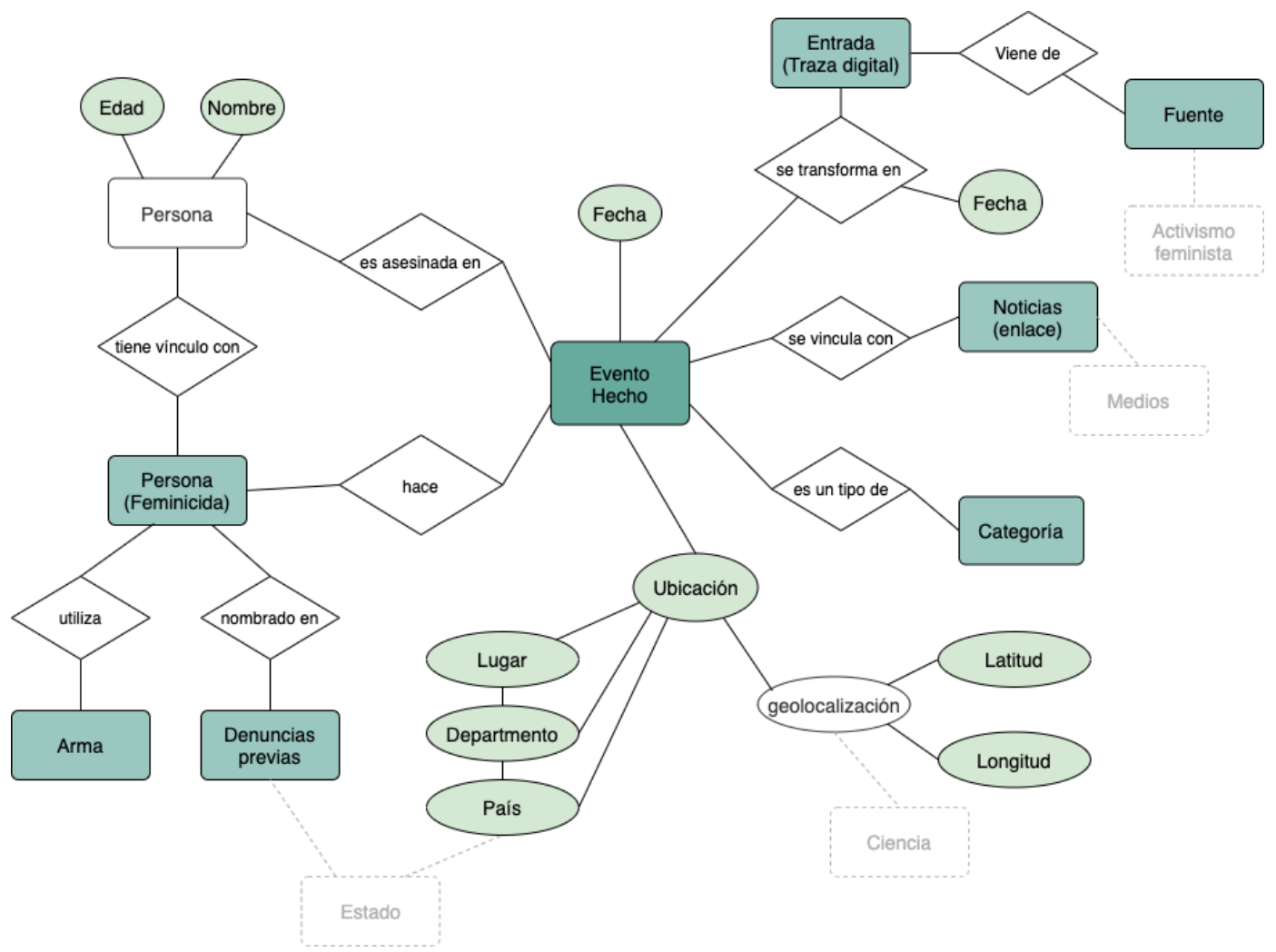

Fuente: elaboración propia.

Hasta aquí, la ontología de datos implica a una persona, una fecha y un lugar, pero ¿cómo se relacionan? Las propiedades ubicación y tiempo parecen enmarcar un evento y la columna denominada "Descripción", que en las tablas de datos de Google Maps se convierte en “¿Cómo la mataron?”, le da más definición con un 
breve texto que delinea la situación estereotipada que presenta este marco de datos. Es un evento o, más precisamente, un Hecho: alguien le hizo algo a alguien, en una fecha determinada, en un lugar dado, de una manera específica. La etiqueta "Feminicida" (“QQuién la mató?” en la tabla de datos del mapa) introduce un actor clave, y una acción. Aquí, el término de género neutro feminicida se utiliza en un sentido paralelo a homicida, e incluso sin tener conocimiento previo del término, se puede intuir a partir de su composición etimológica que refiere a alguien que asesinó a una mujer[16]. Mediante el examen de los valores bajo la etiqueta "Feminicida" vemos que esta persona queda enmarcada completamente en relación con la primera, apareciendo en la escena como pareja o expareja, padre, cliente, sin vínculo, etc. Cabe destacar que es la única etiqueta de columna que referencia directamente el término feminicidio como categoría y al implicar un hacedor de un hecho, un autor de un delito, este descriptor pone en la escena a una segunda Persona: un asesino feminicida.

Acto seguido, el marco introduce una Categoría, cuyos valores se modulan con una serie de calificadores: feminicidio íntimo, transfeminicidio, feminicidiosuicidio, etc. Toledo Vázquez (2009, p. 29) ha señalado que, en la práctica, aun cuando profesan la definición más amplia de feminicidio, que incluye muertes violentas sin autor directo (por ejemplo, muertes por inseguros abortos ilegales), los estudios sobre el tema generalmente se limitan a las muertes violentas en hechos delictivos. Este es el caso aquí: a pesar de la inclusión de algunos casos de suicidio (donde la pareja o expareja de la mujer la sometió a violencia previo al suicidio), el marco de datos da prominencia al estereotipo "acto delictivo", lo cual se amplifica con etiquetas que traen al marco evidencias de un "Arma" y de "Denuncias Previas". Una vez más, se puede percibir la presencia del Estado, ahora en su rol de receptor de denuncias de violencia que sin embargo no impidió un desenlace letal.

Además del Estado, otros actores se ven al fondo en el marco de datos. Las etiquetas "Enlaces" y "Más enlaces" describen una relación con una entidad externa pero accesible (a través de hiperenlaces) desde el marco de datos: las Noticias. Esto dirige la atención a los Medios de Comunicación, que también juegan un papel como agencias de enmarque, ya que las narrativas de los medios 
de comunicación influyen cómo se entiende un tema delictivo, su contexto y posibles soluciones, y estas narrativas a menudo dejan sin explorar las relaciones entre el delito y fuerzas sociales más amplias (Sacco, 1995, p. 153). En líneas similares, las dos últimas etiquetas nombran a una "entrada", evidenciando que hay un proceso mediante el cual las trazas digitales de los casos entran, o más bien se introducen, al marco de datos. Aquí, el marco de datos esboza (difusamente) la presencia histórica de las Activistas feministas como fuente de este trabajo de categorización y rastreo, como enmarcadoras. Por último, cabe señalar una entidad menos prominente pero significativa. Apenas indicada en el marco de datos aparece la Ciencia y Tecnología[17]: la inclusión de coordenadas GPS evoca la cuadrícula global (otro marco) pero también el truco divino de verlo todo desde ningún lado (Haraway, 1991, p. 189). Su presencia da una pátina científica al marco de datos, conjurando la solidez específica que aseguran los reclamos de objetividad de los métodos cuantitativos y su supuesta legitimidad (McLafferty, 1995, p. 436).

¿Cuán cerca convergen el arreglo de hechos en este marco de datos con el feminicidio como categoría? ¿Se puede re-trazar el marco categórico a partir de esta ontología de datos? Visto que la existencia de violencia previa, la relación entre la mujer y su asesino y la forma en que fue asesinada, incluido el entorno, son datos clave para determinar si un caso es un delito de género o un asesinato por motivos diferentes (Tavera Fenollosa, 2008, pp. 333-334; Bernal Sarmiento et al., 2014, p. 92), que el marco de datos presente las denuncias previas, el arma y la relación entre el feminicida y la mujer ayuda a encajar el hecho en el marco de feminicidio. Sin embargo, el género no está presente (no se presenta) explícitamente en el marco de datos, ni como entidad, ni como propiedad de las personas en la escena. Sin lugar a duda, otros marcos conceptuales traerán el género a la lectura, por ejemplo, un marco heteronormativo asumiría el género de la "pareja o expareja" como "masculino", mientras que un marco cisnormativo asumiría que todas las mujeres en la lista son "mujeres biológicas", cuando de hecho se incluyen asesinatos de mujeres trans. Este fue el hallazgo más sorprendente para mí como "enmarcadora" de las trazas digitales en FU. El análisis sugiere que sin una comprensión previa de la categoría feminicidio, sin 
familiaridad con este marco feminista y sus dados ontológicos, este dataset no guiaría la atención directamente al género como identidad y menos aún hacia las desigualdades estructurales de género y las relaciones asimétricas de poder como elementos constitutivos de los asesinato de mujeres allí trazados. En última instancia, dado que la organización y presentación de trazas digitales en este marco de datos no da prominencia al género, no es posible re-trazar completamente el marco categórico de feminicidio -muertes violentas de mujeres por razones de género- a partir de la reconstrucción ontológica.

No obstante, la organización y presentación específicas de hechos, entidades y relaciones en este marco de datos abre ciertas vías de acción. Al igual que sus predecesoras, Crónica de la Violencia (La República de las Mujeres), campaña Crespón negro y Quienes eran (ONG Caminos), FU se basa en una convicción de que hay valor en decir y mostrar el nombre, juntar algunos restos de una vida, visibilizar y reconocer la pérdida públicamente (Butler, 2009, p. 39). Así, nombrar a las mujeres asesinadas materializa y habilita una forma de acción clave: el duelo público como forma de activismo, que se ejemplifica en trabajos que usan los datos como fuente para otras intervenciones activistas, tal como la performance callejera colectiva La Caída de las Campanas, de la artista Hekatherina C. Delgado[18], que se repite en Montevideo después de cada caso. Presentar los datos de esta forma personalizada puede facilitar a los espectadores a hacer conexiones entre los casos y su propia experiencia de vida, orientando su atención hacia algo familiar en el marco y, quizás, hacia la acción.

El marco de datos también habilita a rastrear la complejidad social de la violencia de género y los múltiples factores implicados. Como se mencionó anteriormente, haciendo alusión a su rol enmarcador, los medios aparecen como una entidad vinculada y el marco de datos pone a disposición un archivo de hiperenlaces desde el que se puede iniciar un examen del tratamiento de los casos en los medios de comunicación. Del mismo modo, a partir de este marco de datos se podría trazar el rol del estado, realizando un seguimiento con las instituciones relevantes para investigar la (falta de) intervención en aquellos casos en que la entrada muestra haber denuncias previas. Además, con la salvedad de que es "imperfecto", el dataset podría facilitar el análisis estadístico y visualización de la 
violencia letal contra las mujeres en Uruguay durante un período de casi 20 años, centrándose en las variables de ubicación, fecha, edad, etc. Este tipo de análisis podría ayudar a identificar determinadas zonas o grupos etáreos para dirigir acciones de intervención y apoyo, desde desarrollar iniciativas locales para abordar la violencia por razones de género en la comunidad hasta exigir compromisos del estado para desarrollar políticas públicas o asignar presupuesto. Finalmente, en el enmarque del proyecto como "herramienta de denuncia incompleta e imperfecta" en la página "Acerca de" hay implícito un llamado a la acción a feministas y activistas de datos, una invitación a completar y mejorar este marco de datos.

\subsection{Seguridad y violencia de género}

Hasta hace poco, FU era el único dataset de casos de asesinatos de mujeres por razones de género en Uruguay disponible públicamente, de fácil acceso y posibilidad de reutilización. Si bien el estado ha publicado informes anuales sobre violencia doméstica y homicidios de mujeres desde 2005 y publicó un informe sobre femicidios íntimos para el período 2012-2016 (Gambetta Sacías \& Coraza Ferrari, 2017), estos datos son difíciles de encontrar y solo están disponibles en formato PDF o papel. Por añadidura, casi cada año se ha presentado la información con un desglose diferente (por región, grupo de edad, etc.) al anterior y ningún informe presenta datos específicos sobre los casos, por lo que los datos oficiales no son fácilmente reutilizables o comparables (ver Medina \& da Rosa, 2012).

Las leyes vigentes en Uruguay definen a la violencia femicida como aquella que "causa la muerte de una mujer por el hecho de serlo, o la de sus hijas, hijos u otras personas a su cargo, con el propósito de causarle sufrimiento o daño" (Parlamento del Uruguay, 2018, Ley N. ${ }^{0}$ 19580, Art. 6 N) y al femicidio como el homicidio de una mujer motivado por el odio, desprecio o menosprecio por su condición de tal (Parlamento del Uruguay, 2017, Ley N. ${ }^{\circ}$ 19538, Art. 3). La ley presume motivación por razones de género cuando:

a) A la muerte le hubiera precedido algún incidente de violencia física, psicológica, sexual, económica o de otro tipo, cometido por el autor 
contra la mujer, independientemente de que el hecho haya sido denunciado o no por la víctima

b) La víctima se hubiera negado a establecer o reanudar con el autor una relación de pareja, enamoramiento, afectividad o intimidad.

c) Previo a la muerte de la mujer el autor hubiera cometido contra ella cualquier conducta que atente contra su libertad sexual

(Parlamento del Uruguay, 2017, Ley N.o 19538, Art. 3)

Estas leyes requieren y aportan un marco para que las instituciones estatales recopilen datos sobre violencia contra las mujeres. En marzo de 2019, MI publicó un dataset en el catálogo de datos abiertos del estado titulado Seguridad y violencia basada en género[19]. El dataset completo contiene datos del Sistema de Gestión de Seguridad Pública sobre "violencia doméstica y sus asociados, delitos sexuales, homicidios domésticos a mujeres y femicidios” para el año 2018. Cada conjunto de datos está disponible en forma de tabla (formatos CSV o XLS) y como texto estructurado (formato XML), accesibles, como en FU a través de $<$ iframe $>$. La reconstrucción ontológica que sigue se enfoca en la tabla "Homicidios domésticos a mujeres" (Figura 2). En julio de 2020, luego de concluido este trabajo, se sumó al dataset una tabla titulada "Femicidios" con idéntico arreglo de datos. 


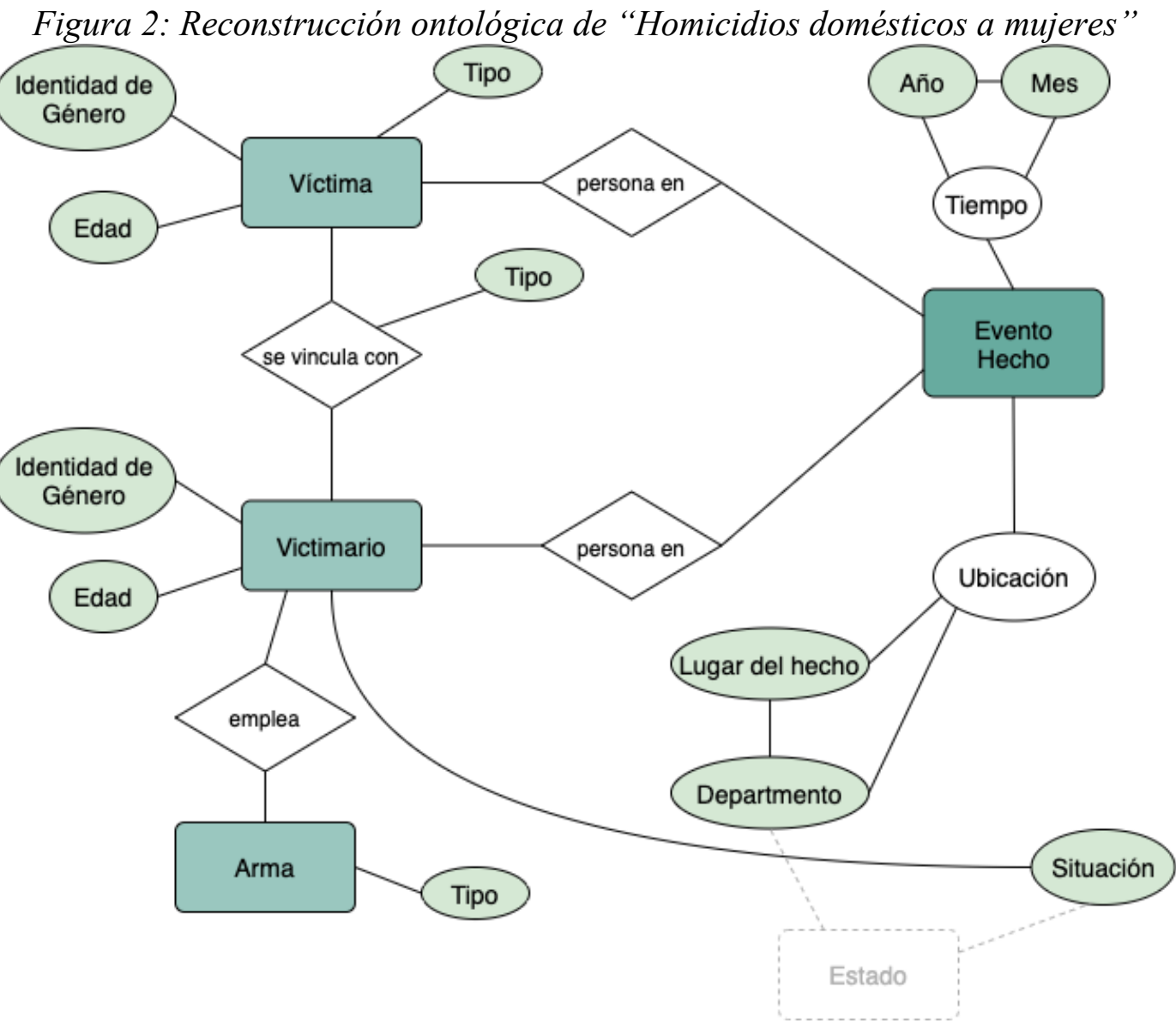

Fuente: elaboración propia.

Las primeras etiquetas en la tabla "Homicidios domésticos a mujeres" fijan el marco temporal y espacial en una escala menos precisa que FU: el tiempo se especifica a nivel de "Mes" y "Año" y la ubicación en el nivel administrativo de "Departamento". La ubicación se caracteriza además en la columna "Lugar del hecho", sus valores describiendo el escenario del homicidio. En este marco de datos, el evento se convoca explícitamente como un Hecho que implica un arma y dos personas presentadas a través de las propiedades "Identidad de género" y "Edad": una Víctima y un Victimario. La noción de "identidad de género" aparece en la escena entendida como "la percepción que una persona tiene sobre su género, relevado de la información aportada por familiares, testigos, etc.” según los metadatos del dataset. Aunque se establecen otros tipos de relaciones al describir lazos afectivos u otros ("Tipo de vínculo") o si las mujeres eran el objetivo directo de los agresores ("Tipo de víctima"), en este marco la insistencia en las etiquetas víctima/victimario (repetidas en seis de las columnas) encierra a estas personas en una aparentemente autónoma dicotomía fatal. Ontológica y 
políticamente esta caracterización puede esencializar a las personas en el marco, que en esta versión de los "hechos" podrían percibirse como portadoras de propiedades inherentes que las separan de otras "mujeres" (no-víctimas) y otros "hombres" (no-victimarios). Por último, el Estado se presenta en este marco de datos, al igual que en FU, a través de la división administrativa de departamentos, pero también es visible en su rol de administrador de justicia, cuando la etiqueta "Situación del victimario" muestra a los "victimarios" ingresando al proceso judicial.

¿Qué tan precisamente convergen la organización y presentación de hechos en este marco de datos con la categoría feminicidio, aquí nombrada femicidio? Cabe destacar que este marco de datos no presenta ninguno de los tres elementos señalados en la propia ley para sustentar la presunción de motivación de género: existencia previa de violencia, el "No" de la víctima a una relación afectiva con el agresor, o un elemento sexual. De hecho, a pesar de la mención de la categoría femicidio en la página principal, inicialmente el dataset solo contenía la tabla titulada "Homicidios domésticos a mujeres" (recién en 2020 aparece la tabla "Femicidios"). Este desplazamiento terminológico probablemente sea reflejo de tensiones tecnológicas y legales derivadas de la traducción y reciente introducción de la categoría femicidio como figura penal. "Homicidios domésticos" convoca un marco legal previamente establecido que designa como "violencia doméstica" a la violencia por parte de una pareja, conviviente o familiar (Parlamento del Uruguay, 2002, Ley N. ${ }^{\circ}$ 17514). Las activistas feministas que pugnaron por esta ley han descrito su formulación de género neutro como "un defecto de nacimiento [producto de] duras negociaciones políticas con un sistema legislativo desconocedor de la problemática de género" y reacio a aprender (Jubin \& Samuniski, 2013, p. 28). La coexistencia en el dataset de ambos marcos categóricos evidencia estas controversias penales y llama la atención a comparar la relación con el género (o no) de los homicidios de mujeres.

Las descripciones de entidades y propiedades que ofrece este marco de datos hacen posible una gama más limitada de acciones que FU. A diferencia del dataset activista, el anonimato de las personas que se presentan en este marco de datos no facilita acciones que conecten los datos directamente con la experiencia de vida, 
tal como el duelo público mencionado más arriba. También cierra la posibilidad de seguir rastreando las trazas, por ejemplo, haciendo seguimiento de los casos después que ingresan al proceso judicial. El análisis estadístico y la visualización de datos parecen ser las únicas posibles vías para la acción que abre este dataset, limitados al marco temporal del dataset, que es tan sólo un año. Es de esperar que el estado continúe publicando datos abiertos (aunque aún no lo ha hecho) y será interesante ver si futuras versiones mantienen o modifican la organización actual de las trazas digitales.

\section{Conclusiones}

Existen varias diferencias entre los datasets de asesinatos de mujeres por razones de género de FU y MI: desde las personas involucradas en su producción, con sus diferentes pertenencias institucionales, motivaciones y orientaciones afectivas, pasando los métodos para recopilar datos (monitoreo de medios el primero; informes policiales y sentencias judiciales el segundo), los soportes y plataformas a través de los cuales publican los datos (sitio web y redes sociales; portal del estado) y sus formatos (Google Maps y Google Spreadsheets; archivos XML o CSV). Pero crucialmente, diferentes definiciones específicas de la violencia letal por razones género, que forman parte del conocimiento de dominio de quienes diseñan las tablas, organizan las maneras en que se arreglan las trazas digitales de los eventos. En cada tabla, diferentes entidades y relaciones, descritas de maneras diferentes, se encajan al marco del asesinato por razones género con distintas descripciones, dando lugar a distintas versiones de los casos y habilitando ciertas acciones (y no otras). Es decir, las diferencias entre los datasets son tecnológicas y metodológicas, pero también políticas y ontológicas.

Cada vez más activistas y estados de América Latina están recopilando y publicando datos sobre asesinatos de mujeres por razones de género, enmarcados en variantes de la categoría feminicidio, arreglos de datos que tienen efectos políticos, afectivos y éticos. Considerando importante entender estos efectos, este estudio ha puesto el foco en las tablas, o marcos de datos, donde se configuran las trazas digitales de la violencia, realizando una reconstrucción ontológica de los datasets producidos por FU y MI. En el análisis, el feminicidio como categoría se 
ha revelado como un marco categórico que guía a quienes diseñan datasets, tanto durante la investigación de eventos saturados de género como durante el diseño del arreglo de datos. A su vez, los marcos de datos que constituyen estos arreglos dan prominencia (o realizan) a entidades y propiedades específicas y así guían la atención, estrechando algunas vías para la comprensión y la acción y ensanchando otras (Walby et al., 2017, p. 11). El objetivo de este trabajo ha sido retrazar los marcos categóricos de cada dataset mediante la reconstrucción de sus respectivas ontologías de datos, mostrando qué entidades y relaciones surgen en cada instancia y las diferentes acciones que sus descripciones posibilitan.

¿Se puede inferir los marcos categóricos que sostienen la creación datasets de asesinatos de mujeres por razones género a partir de una reconstrucción ontológica? No del todo. Tanto FU como MI afirman construir sus datasets desde marcos categóricos donde el género constituye un elemento fundamental, ya sea feminicidio o femicidio. Sin embargo, el análisis encontró que ni FU ni el dataset de MI ofrecen descripciones que den prominencia o dirijan la atención explícitamente hacia el elemento social de las relaciones de género o el diferencial de poder entre hombres y mujeres. Como Bowker y Star (1999, p. 101) han señalado, el diseño de ontologías de datos nunca puede ser más que una operación inicial y contingente, en el sentido de que en último análisis siempre es posible actuar de otras maneras, esculpir el mundo de formas diferentes. Sin embargo, esta operación de diseño se podría refinar para lograr una mayor convergencia entre los eventos y el enmarque categórico que sostiene los marcos de datos donde se presentan las eventuales trazas digitales.

Por ejemplo, implicar a entidades (supuestamente) externas el evento, como ser los medios de comunicación, el estado y las activistas feministas, es un gesto hacia la naturaleza social de los asesinatos por razones el género, ya que sugiere, como indica Fraser (2010, p. 73), que lo que ocurre en un contexto particular no se puede explicar o justificar únicamente por este contexto, ni por las entidades físicas que la componen, ni se pueden reducir contexto en sí. Revisar y modificar las casillas y etiquetas en que se organizan y presentan las trazas digitales de asesinatos de mujeres podría hacer más evidente la implicación de todas las entidades y relaciones que un marco categórico dado conecta al evento. Esta 
revisión debería incluir un análisis del enmarque afectivo de cada marco de datos, un aspecto poco explorado en este trabajo por limitaciones de espacio: ¿cómo guían los distintos arreglos de datos las orientaciones afectivas y emocionales hacia los asesinatos por razones de género, por ejemplo, cuando se presenta a las mujeres como personas nombradas versus víctimas anónimas? Otra posible mejora sería intentar dar más definición dentro del marco de datos a otras dimensiones de género, yendo más allá de la identidad de género y la relación entre la mujer y su asesino. Por ejemplo, se podría agregar o subrayar algunas pistas sobre la motivación de género, con los elementos definidos en la ley uruguaya para garantizar a las mujeres una vida libre de violencia basada en género (Ley N. ${ }^{\circ}$ 19580) y en el Modelo de protocolo latinoamericano de investigación de las muertes violentas de mujeres por razones de género (femicidio/feminicidio) de la ONU como puntos de partida.

Para el período 2012-2016, MI informó que el 54,2\% de todos los homicidios de mujeres en Uruguay fueron homicidios íntimos, en comparación con el 3,3\% para los hombres (Gambetta Sacías \& Coraza Ferrari, 2017, p. 31). Como han demostrado Walby y colegas (2017, p. 5), el análisis de la naturaleza de género de la violencia requiere comparaciones entre mujeres y hombres, que no son posibles si el enfoque analítico y los datos recogidos refieren sólo a las mujeres. Tanto FU como MI presentan a los asesinatos de mujeres por razones de género por separado, lo cual esconde la asimetría de género que resultaría más evidente situando a los feminicidio en relación a todas las formas de violencia letal. Además, si bien la identidad de género, incluyendo a mujeres trans, está presente en ambos marcos de datos (más débilmente en FU) y FU señala el estado migratorio (en el espacio para "Otras informaciones"), ni el dataset activista ni el del estado registran trazas de raza/etnia, orientación sexual, clase u otras dimensiones que podrían permitir análisis interseccionales de la violencia letal de género en Uruguay. Aparte de cerrar la vía para investigar cómo están implicados estos elementos, esta falta contribuye a crear un efecto homogeneizador y esencializante, especialmente sobre las mujeres, que además quedan fijas en el papel de víctimas (más en el dataset de MI), pero también sobre cada asesinato, que es en última instancia único y particular. Este aspecto es crítico, ya que lo que 
está en juego en estos marcos de datos no es únicamente, como señala Mol (1999, p. 86), cómo se representa la realidad en información que circula como palabras e imágenes, sino también la configuración material de la realidad que se realiza en diagnósticos, intervenciones y prácticas de investigación.

Este trabajo se ha centrado en tablas, o marcos de datos, entendiendo que están implicados en estructuras de datos más complejas. De interés para futuras investigaciones sería examinar cómo se (re)configuran las trazas digitales de asesinatos de mujeres por razones género al circular en y a través de otras estructuras de datos. Por ejemplo, cuando los datos se comparten entre instituciones estatales, cuando otros actores de la sociedad civil descargan y reutilizan los datos, o en el movimiento de las trazas digitales desde una tabla a un mapa o a otras formas de visualización, que luego circulan en redes sociales.

Las nociones de feminicidio como marco, trazas digitales de asesinato y marco de datos resultan prismas útiles para analizar los datos sobre homicidios por razones género. Queda más por hacer, pero concluyo aquí, esperando que este trabajo aporte un enfoque original al estudio crítico de los datos, pero más que nada, que los análisis, ideas y recomendaciones en estas páginas sirvan para apoyar las prácticas de datos activistas y estados que usan estos marcos para abordar, y un día poner fin, a la violencia de género contra las mujeres.

\section{Referencias bibliográficas}

Bento, B. (2014, junio 4). Brasil: Pais do Transfeminicídio. Centro LatinoAmericano em Sexualidade e Direitos Humanos. Recuperado de http://www.clam.org.br/uploads/arquivo/Transfeminicidio_Berenice_Bent o.pdf

Berkins, L. (2015, junio 12). El travesticidio también es femicidio. Página 12.

Recuperado de https:/www.pagina12.com.ar/diario/suplementos/las12/139791-2015-06-12.html

Bernal Sarmiento, C., Lorente Acosta, M., Roth, F., \& Zambrano, M. (2014). Modelo de protocolo latinoamericano de investigación de las muertes violentas de mujeres por razones de género (femicidio/feminicidio). ONU Mujeres; OACNUDH. 
Boira, S., Marcuello-Servós, C., Otero, L., Sanz Barbero, B. (2015). Femicidio y feminicidio: Un análisis de las aportaciones en clave iberoamericana. Comunitania: International Journal of Social Work and Social Sciences, 10, 27-46.

Bowker, G. C., Star, S. L. (1999). Sorting things out: Classification and its consequences. MIT Press.

Bueno-Hansen, P. (2011). Feminicidio: El aprovechamiento al máximo de un «termino empoderado». En: R.-L. Fregoso, C. Bejarano, \& M. Lagarde y de los Ríos (Eds.), G. Trevisan (Trad.), Feminicidio en América Latina. Universidad Nacional Autónoma de México, Centro de Investigaciones Interdisciplinarias en Ciencias y Humanidades : Red de Investigadoras por la Vida y la Libertad de las Mujeres.

Butler, J. (2009). Frames of War: When is Life Grievable? Verso.

Chen, P. P. (1976). The entity-relationship model: Toward a unified View of data. ACM Transactions on Database Systems.

Chenou, J.-M., Cepeda-Másmela, C. (2019). \#NiUnaMenos: Data Activism From the Global South. Television \& New Media, 20, (4), 396-411.

Coleman, R., Banning, S. (2006). Network TV News' Affective Framing of the Presidential Candidates: Evidence for a Second-Level Agenda-Setting Effect through Visual Framing. Journalism \& Mass Communication Quarterly, 83, (2), 313-328.

Dalton, C., Thatcher, J. (2014, mayo 12). What does a critical data studies look like, and why do we care? Society \& Space. Recuperado de https://societyandspace.org/2014/05/12/what-does-a-critical-data-studieslook-like-and-why-do-we-care-craig-dalton-and-jim-thatcher/

Dawson, M., Carrigan, M. (2020). Identifying femicide locally and globally: Understanding the utility and accessibility of sex/gender-related motives and indicators. Current Sociology, 1-23.

de Vos, M. (2014). Interpreting environmental computational spreadsheets. En: P. Grothand N. Noy (Eds.), Proceedings of the Doctoral Consortium at the 13th International Semantic Web Conference (ISWC 2014).

de Vos, M., van Hage, W. R., Ros, J., Schreiber, G. (2012). Reconstructing Semantics of Scientific Models: A Case Study. Proceedings of the OEDW Workshop on Ontology Engineering in a Data Driven World. Galway, Ireland: EKAW. 
Deus, A., González, D. (2018). Análisis de Legislación sobre Femicidio/Feminicidio en América Latina y el Caribe e Insumos para una Ley Modelo. MESECVI; ONU Mujeres.

D’Ignazio, C., Klein, L. F. (2020). Data feminism. The MIT Press.

Engle Merry, S., Bibler Coutin, S. (2014). Technologies of Truth in the Anthropology of Conflict: Gender Violence and the Social Construction of Reality. zeitschrift für menschenrechte, Menschenrechte und Gewalt, 2848.

Entman, R. M. (1993). Framing: Toward Clarification of a Fractured Paradigm. Journal of Communication, 43, (4), 51-58.

Flyverbom, M., Murray, J. (2018). Datastructuring—Organizing and curating digital traces into action. Big Data \& Society, 5, (2).

Fraser, M. (2010). Facts, Ethics and Event. En: C. B. Jensen, K. Rödje (Eds.), Deleuzian Intersections: Science, Technology, Anthropology. Berghahn Books. (pp. 57-82).

Fumega, S. (2019). Open data, gender and violence in Latin America. European Public Mosaic, 7, 36-47.

Gambetta Sacías, V., Coraza Ferrari, P. (2017). Femicidios íntimos en Uruguay Homicidios a mujeres a manos de (ex) parejas. Ministerio del Interior.

Gitelman, L., Jackson, V. (2013). Introduction. En L. Gitelman (Ed.), “Raw Data” Is an Oxymoron MIT Press. (pp. 1-14).

Goffman, E. (1986). Frame Analysis: An Essay on the Organization of Experience. Northeastern University Press. (Original 1974)

Hacking, I. (1991). The Making and Molding of Child Abuse. Critical Inquiry, 17, (2), 253-288.

Hacking, I. (1999). The social construction of what?. Harvard University Press.

Hacking, I. (2002). Making Up People. En: Historical ontology. Harvard University Press. (Original 1983) (pp. 99-114).

Haraway, D. J. (1991). Simians, cyborgs, and women: The reinvention of nature. Routledge.

ILDA. (2019). Guía para protocolizar procesos de identificación de femicidios para su posterior registro. ILDA. 
Jubin, M., Samuniski, F. (2013). Violencia basada en género. RUCVDS.

Kitchin, R., Lauriault, T. P. (2014). Towards critical data studies: Charting and unpacking data assemblages and their work (The Programmable City Working Paper N. ${ }^{\circ}$ 2).

Klein, G., Moon, B., Hoffman, R. R. (2006). Making Sense of Sensemaking 2: A Macrocognitive Model. IEEE Intelligent Systems, 21, (5), 88-92.

Labastie, M. (2007). ¡Ni una muerte más por violencia doméstica! Evaluando una campaña. Recuperado de http://www.mujerahora.org.uy/campania_crespo.pdf

Lagarde y de los Ríos, M. (2008). Antropología, feminismo y política: Violencia feminicida y derechos humanos de las mujeres. En: M. Bullen \& C. Diez Mintegui (Eds.), Retos Teóricos y Nuevas Prácticas. Ankulegi. (pp. 209239).

Lagarde y de los Ríos, M. (2010). Preface: Feminist Keys for Understanding Feminicide: Theoretical, Political and Legal Construction. En: R.-L. Fregoso, C. Bejarano (Eds.), Terrorizing Women: Feminicide in the Americas. Duke University Press.

La República de las Mujeres. (2018, agosto 12). 1988-1990: Los primeros pasos: Nombrar y mostrar. Diario La República. Recuperado de https://www.republica.com.uy/1988-1990-los-primeros-pasos-nombrar-y$\underline{\text { mostrar-id670561/ }}$

La República de las Mujeres. (2018, agosto 12). 2005-2009: Un quinquenio que no conformó. Diario La República. Recuperado de https://www.republica.com.uy/2005-2009-un-quinquenio-que-noconformo-id670571/

Luján Pinelo, A. (2018). A Theoretical Approach to the Concept of Femi(ni)cide. Philosophical Journal of Conflict and Violence, 2, (1), 41-63.

Maffía, D. H. (2016, noviembre 30). Crímenes de género: Del femicidio al travesticidio / transfemicidio. Jornada de capacitación sobre "Crímenes de género: del femicidio al travesticidio / transfemicidio", Oficina de la Mujer de la Corte Suprema de Justicia de la Nación, Buenos Aires, Argentina. Recuperado de https://youtu.be/b936aFQpWvg

Manjoo, R. (2012). A/HRC/20/16 Report of the Special Rapporteur on violence against women, its causes and consequences. UN. 
McLafferty, S. L. (1995). Counting for Women. The Professional Geographer, 47, (4), 436-442.

Medina, R., da Rosa, T. (2012). Información pública sobre la intervención del Ministerio del Interior en situaciones de violencia doméstica («Más información. Mejor Prevención.» Acceso a la Información Pública sobre Violencia Doméstica en Uruguay). CAINFO y RUCVDS.

Milan, S., Velden, L. van der. (2016). The Alternative Epistemologies of Data Activism. Digital Culture \& Society, 2, (2).

Minsky, M. (1974). A Framework for Representing Knowledge (MIT-AI Laboratory Memo 306 N. ${ }^{\circ}$ 306).

Mol, A. (1999). Ontological Politics. A Word and Some Questions. The Sociological Review, 47, (1_suppl), 74-89.

Monárrez Fragoso, J. E. (2009). Trama de una injusticia: Feminicidio sexual sistémico en Ciudad Juárez. Colegio de la Frontera Norte.

Monárrez Fragoso, J. E. (2018). Feminicide: Impunity for the Perpetrators and Injustice for the Victims. En: K. Carrington, R. Hogg, J. Scott, M. Sozzo (Eds.), The Palgrave Handbook of Criminology and the Global South (pp. 913-929). Springer International.

Ni Una Menos. (2016, octubre 20). Nosotras paramos [Facebook]. Recuperado de https://www.facebook.com/notes/ni-una-menos/nosotrasparamos $/ 544964935694693$

Osborne, R. (2008). De la «violencia» (de género) a las «cifras de la violencia»: Una cuestión política. Empiria. Revista de metodología de ciencias sociales, 0, (15), 99-124.

Pan, Z., Kosicki, G. M. (1993). Framing analysis: An approach to news discourse. Political Communication, 10, 55-75.

Parlamento del Uruguay. (2002). Violencia Doméstica (Ley N. $\left.{ }^{\circ} 17514\right)$. Parlamento del Uruguay. Recuperado de https://parlamento.gub.uy/documentosyleyes/leyes/ley/17514

Parlamento del Uruguay. (2017). Actos de discriminación y femicidio (Ley N. ${ }^{\circ}$ 19538). Parlamento del Uruguay. Recuperado de https://parlamento.gub.uy/documentosyleyes/leyes/ley/19538 
Parlamento del Uruguay. (2018). Violencia hacia las mujeres basada en género (Ley N. ${ }^{\circ}$ 19580). Parlamento del Uruguay. Recuperado de https://parlamento.gub.uy/documentosyleyes/leyes/ley/19580

Pine, K. H., Liboiron, M. (2015). The Politics of Measurement and Action. Proceedings of the 33rd Annual ACM Conference on Human Factors in Computing Systems - CHI '15, 3147-3156.

Poster, M. (1995). Databases as Discourse; or, Electronic Interpellations. En: The Second Media Age (pp. 78-94).

Radford, J., \& Russell, D. E. H. (1992). Femicide: The politics of woman killing. Twayne.

Reigeluth, T. B. (2014). Why data is not enough: Digital traces as control of self and self-control. Surveillance \& Society, 12, (2), 243-254.

Ruíz, D., Garrido, A. (2018). Rompiendo Moldes: Transformar imaginarios y normas sociales para eliminar la violencia contra las mujeres. Oxfam Internacional.

Russell, D. E. H. (2012, noviembre 26). Defining Femicide. Introductory speech presented to the United Nations Symposium on Femicide. Recuperado de http://www.dianarussell.com/f/Defining_Femicide _United_Nations_Speech_by_Diana_E._H._Russell_Ph.D.pdf

Sacco, V. F. (1995). Media Constructions of Crime. The ANNALS of the American Academy of Political and Social Science, 539, (1), 141-154.

Segato, R. L. (2004). Territorio, soberanía y crímenes de segundo Estado: La escritura en el cuerpo de las mujeres asesinadas en Ciudad Juárez. Departamento de Antropologia, Universidade de Brasília.

Segato, R. L. (2011, mayo 4). Femigenocidio y feminicidio: Una propuesta de tipificación. Mesa "Feminismos Poscoloniales y Descoloniales: Otras Epistemologias”. II Encuentro Mesoamericano de Estudios de Género y Feminismos, Ciudad de Guatemala. Recuperado de https://herramienta.com.ar/articulo.php?id=1687

Solyszko Gomes, I. (2016). Femicidio y feminicidio: Avances para nombrar la expresión letal de la violencia de género contra las mujeres. GénErơ ôs, 20, (13), 23-42.

Stengers, I. (2000). The invention of modern science. (D. W. Smith, Trad.) Vol. 19. University of Minnesota Press. 
Stengers, I., Ralet, O. (1997). Drugs: Ethical Choice or Moral Consensus. En: I. Stengers (Ed.), P. Bains (Trad.). Power and invention: Situating science University of Minnesota Press. pp. 215-232.

Suárez Val, H. (2017). Affect amplifiers: Feminicide, feminist activists and the politics of counting and mapping gender-related murders of women [tesis de maestría]. Goldsmiths, University of London.

Suárez Val, H. (2020). Datos discordantes. Información pública sobre femicidio en Uruguay. Mundos Plurales, 7, (1).

Tavera Fenollosa, L. (2008). Estadísticas sobre violencia de género: Una mirada crítica desde el feminicidio. En: G. Zaremberg, Políticas sociales y género: Los problemas sociales y metodológicos: Vol. II (1. ${ }^{\mathrm{a}} \mathrm{ed} ., \mathrm{pp} .301-$ 345). FLACSO-México.

Toledo Vásquez, P. (2009). Feminicidio: Consultoría para la Oficina en México del Alto Comisionado de las Naciones Unidas para los Derechos Humanos. OHCHR.

Vives-Cases, C., Goicolea, I., Hernández, A., Sanz-Barbero, B., Gill, A. K., et al (2016). Expert Opinions on Improving Femicide Data Collection across Europe: A Concept Mapping Study. PLoS ONE, 11, (2).

Walby, S., Towers, J., Balderston, S., Corradi, C., Francis, B., Heiskanen, M., et al. (2017). The Concept and Measurement of Violence Against Women and Men. Policy Press.

Weil, S., Corradi, C., Naudi, M. (2018). Femicide across Europe: Theory, research and prevention. Policy Press.

\section{Notas}

[1] "Curar" se utiliza como traducción de la voz inglesa "curate", que refiere a la selección, organización, presentación, cuidado y preservación de una muestra artística, en este caso, de datos de feminicidio.

[2] La expresión "raw data" (datos crudos) refiere a datos que supuestamente no están procesados.

[3] "Arreglar" toma aquí los sentidos de "disponer", "clasificar" y de "resolver" o "reparar", que integra la expresión "sort out" que utilizan Bowker y Star. 
[4] Hacking utiliza la expresión "make up" que significa "constituir" e incluye las acepciones "hacer", "inventar", “construir", “componer".

[5] Varias autoras reseñan las diversas posiciones dentro del movimiento feminista y en el ámbito legal (Boira et al., 2015; Deus \& González, 2018, pp. 2225; Solyszko Gomes, 2016; Toledo Vásquez, 2009, pp. 23-34). Algunos de los aspectos más disputados incluyen: si la categoría debe designar solo a los asesinatos intencionales o incluir todas las muertes violentas misóginas y por razones de género (Toledo Vásquez, 2009, p. 26); si solo los hombres pueden cometer feminicidio (Toledo Vásquez, 2009, pp. 15, 77); o si la noción de impunidad debe ser parte del concepto, como la integra Lagarde (2010, p. xv) para señalar la responsabilidad del Estado, o simplemente ser considerada parte del contexto (ver Russell, 2012).

[6] Ver también Hacking (1991, p. 253) y Bowker y Star (1999, p. 254).

[7] Si bien en castellano se utiliza el término "huella digital" para nombrar los registros y datos que dejamos al navegar Internet, elegí el término "traza" por su cercanía etimológica al inglés "trace" y el francés "trace" de los trabajos citados.

[8] Si bien hay mujeres en los organismos del estado que producen datos oficiales, uso el masculino para recordar que el estado representa el poder patriarcal.

[9] Entman (1993, p. 54) expresa una idea similar, al decir que los marcos seleccionan y llaman la atención sobre aspectos particulares de la realidad descrita, lo que lógicamente significa que simultáneamente desvían la atención de otros aspectos.

\section{[10] http://caminos.org.uy/quieneseran.html}

[11] http://zeemaps.com

[12] http://feminicidiouruguay.net

[13] http://facebook.com/feminicidio.uruguay (desde noviembre 2016)

[14] http://twitter.com/feminicidioURY (desde febrero 2017)

[15] http://instagram.com/feminicidiouruguay (desde febrero 2018) 
[16] Sobre la etimología de la palabra, consultar (Monárrez Fragoso, 2009, p. 44; in Luján Pinelo, 2018, pp. 34-35).

[17] Si bien la presencia de la tecnología debería ser evidente en un formato digital, su ubiquidad la torna invisible.

[18] En los años en que se desarrolló esta intervención pública (2015-2018), Hekatherina solía utilizar FU para buscar casos y viceversa: a veces me enteraba de un caso cuando aparecía en mi muro de Facebook el llamado a participantes para una nueva Caída. https://catalogodatos.gub.uy/dataset/tic-ministerio-del-interiorseguridad_y_violencia_en_genero_ministerio_interior

\section{Nota contribución autoral}

La totalidad del trabajo estuvo a cargo de Helena Suárez Val.

\section{Nota del editor}

El editor responsable para la publicación de este artículo fue Mario Barité. 